\title{
Análise fatorial da adsorção de cobre em solução aquosa por hidroxiapatita
}

\author{
Factor analysis of copper adsorption in aqueous solution by hydroxyapatite
}

\author{
Dany Geraldo Kramer* ${ }^{1 *}$, Geraldo Barroso Cavalcanti Junior ${ }^{1} \odot$, \\ Anesio Mendes de Sousa' ${ }^{\oplus}$, João Batista Costa' ${ }^{\odot}$
}

口-

\section{RESUMO}

O cobre (Cu) é um contaminante frequente das águas residuais, o que pode levar a intoxicação humana e danos ambientais. A hidroxiapatita (HAP) pode ser utilizada para remoção dessa substância de efluentes, pois é de fácil produção e alto rendimento. Assim, o presente estudo objetivou analisar a influência de vários fatores (factorial design) na adsorção do $\mathrm{Cu}^{+2}$ em solução. A HAP foi produzida por meio de precipitação aquosa e, subsequentemente, caracterização por difração de raios $X(D R X)$, espectroscopia no infravermelho por transformada de Fourier (FTIR), espectroscopia por energia dispersiva (EDS) e microscopia eletrônica de transmissão (TEM). A influência das variáveis pH, concentração inicial (Ci) e massa adsorvente na adsorção de $\mathrm{Cu}^{+2}$ foi analisada por intermédio de um planejamento fatorial $12\left(2^{3}\right)$, com 4 pontos centrais. As três variáveis analisadas foram estatisticamente significantes, sendo possível observar remoções acima de 80\% do metal dissolvido em solução. A análise de variância (ANOVA) mostrou que o pH da solução (1), a massa adsorvente (3), a concentração inicial de metal (2) e a interação $2 \times 3$ (2³) foram estatisticamente significantes. A eficiência máxima de remoção de $\mathrm{Cu}^{+2}$ obtida no procedimento de otimização foi de 85,33\%.

Palavras-chave: adsorção; cobre; hidroxiapatita; fatorial; solução; análise.

\begin{abstract}
Copper is a frequent contaminant of wastewater, which can lead to human intoxication and environmental damage. The hydroxyapatite (HAP) can be used to remove this substance from wastewater, as it is easy to produce and has high yield. Thus, the present study aimed to analyze the influence of several factors (factorial design) on the adsorption of $\mathrm{Cu}^{+2}$ in solution. HAP was produced by means of aqueous precipitation and subsequently characterized by XRD, FTIR, EDS, and TEM. The influence of the variables $\mathrm{pH}$, initial concentration (Ci), and adsorbent mass on the adsorption of $\mathrm{Cu}^{+2}$ was performed through 12 factorial design $\left(2^{3}\right)$, with four central points. The three variables analyzed were statistically significant, being possible to observe removals above $80 \%$ of the dissolved metal in solution. The analysis of variance (ANOVA) showed that the $\mathrm{pH}$ of the solution (1), the adsorbent mass (3), the initial concentration of metal (2), and the interaction $2 \times 3$ were statistically significant. The maximum $\mathrm{Cu}^{+2}$ removal efficiency obtained in the optimization procedure was $85.33 \%$.
\end{abstract}

Keywords: adsorption; copper; hydroxyapatite; factorial; solution; analysis.

\section{INTRODUÇÃO}

O cobre $(\mathrm{Cu})$ é um metal pesado que pode ser liberado no meio ambiente por meio da mineração, do setor industrial (refinarias de petróleo, cimento, concreto e produção de papel) e das indústrias de cerâmica e vidro, levando a riscos às saúdes humana e ambiental (CHENG et al., 2010; SHENG et al., 2010, DA'NA; SAYARI, 2011; KUMAR et al., 2011; CÓRDOVA et al., 2012; ROSSKOPFOVA et al., 2012, KYZAS et al., 2013).

As técnicas de remoção de metais pesados têm sido investigadas nos últimos anos, como precipitação química, troca iônica, osmose reversa e adsorção. A adsorção tem vantagens por sua eficiência, facilidade de manuseio e aplicabilidade de adsorventes de baixo custo (CHENG et al., 2010; DA'NA; SAYARI, 2011; SANTOS et al., 2011; CÓRDOVA et al., 2012; ROSSKOPFOVA et al., 2012).

Bagaço de cana (SANTOS et al., 2011), sílica (DA’NA; SAYARI, 2011), castanha de caju (KUMAR et al., 2011), folhas de árvore triturada (CHEN et al., 2010) e HAP em pó (GHANDHI; KOUSALYAB; MENAKSHI, 2011; LIANG et al., 2011; ROSSKOPFOVA et al., 2012) são exemplos de materiais relatados na literatura para a remoção de $\mathrm{Cu}$ por adsorção.

A HAP tem ocorrência natural em ossos e dentes de mamíferos. Sua utilização na remoção de metais pesados de efluentes é justificada, pois apresenta fácil produção por meio da técnica de precipitação aquosa e alto rendimento na remoção desses elementos químicos (NAYAK, 2010; GHANDHI; KOUSALYAB; MENAKSHI, 2011; LIANG et al., 2011; ROSSKOPFOVA et al., 2012).

Os métodos clássicos de estudo para a remoção de $\mathrm{Cu}$ por HAP ocorrem levando-se em consideração a definição de fator em um determinado período. 
Esses estudos são mais longos e requerem maior número de experimentos para encontrar as condições ideais para a aplicação da massa adsorvente. Além disso, não permitem uma avaliação do efeito combinado de diversas variáveis no processo de adsorção (FERNAME et al., 2010; NAYAK, 2010; ROSSKOPFOVA et al., 2012).

O factorial design é uma ferramenta útil para estudo multifatorial, na qual a interação entre diversas variáveis pode ser analisada por modelos estatísticos, indicando as melhores condições experimentais em menor tempo e custo de operação (DA'NA; SAYARI, 2011; CÓRDOVA et al., 2012). Dessa forma, o presente estudo teve como objetivo analisar a influência de diversos fatores no processo de adsorção de $\mathrm{Cu}^{+2}$ por HAP.

\section{METODOLOGIA}

\section{Síntese da hidroxiapatita}

A HAP foi sintetizada por meio da técnica de precipitação aquosa, devido ao seu baixo custo e a sua simplicidade operacional. A Equação 1 ilustra a rota dessa síntese pela adição de grupos fosfato aos íons de cálcio suspensos (ESLAMI et al., 2010; NAYAK, 2010; MONDAL et al., 2012).

$(10-\mathrm{x}) \mathrm{Ca}(\mathrm{OH})_{2}+6 \mathrm{H}_{3} \mathrm{PO}_{4} \rightarrow \mathrm{Ca}_{10}-\mathrm{x}\left(\mathrm{HPO}_{4}\right)_{\mathrm{x}}\left(\mathrm{PO}_{4}\right)_{6}-\mathrm{X}+18-\mathrm{X}\left(\mathrm{H}_{2} \mathrm{O}\right)$

A suspensão de $\mathrm{Ca}(\mathrm{OH})_{2}(0,5 \mathrm{M})$ foi preparada por agitação e aquecimento (1 hora $\left./ 50^{\circ} \mathrm{C}\right)$, seguida pela adição de $\mathrm{H}_{3} \mathrm{PO}(0.3 \mathrm{M})$, garantindo uma relação $\mathrm{Ca} / \mathrm{P}$ de 1,67. A reação de neutralização ocorreu nas seguintes condições: taxa de gotejamento ( 2 gotas/s), temperatura a $80^{\circ} \mathrm{C}$, vigorosa agitação e $\mathrm{pH}>9,0$ (adição de hidróxido de amônio) para reduzir subprodutos indesejáveis.

Ao final do gotejamento, o material obtido da reação foi deixado em repouso para maturação (12 horas), gerando, ao final, um precipitado branco. Esse material foi filtrado e lavado com água desionizada e ácido fosfórico $(0,1 \mathrm{mmol} / \mathrm{L})$, para remover os íons residuais de amônio e cálcio. Finalmente, o material foi seco em estufa $\left(100^{\circ} \mathrm{C} / 12\right.$ horas) e desagregado com pistilo de ágata, cujo pó resultante foi calcinado a $700^{\circ} \mathrm{C}$ por 1 hora (FDG-7000).

\section{Técnicas de caracterização da hidroxiapatita}

A estrutura cristalina da HAP sintetizada foi analisada por difração de raios X (XRD - Rigaku, modelo Miniflex II), operando nas condições 30kV e $15 \mathrm{~mA}$, ânodo de Cu, $\mathrm{K} \alpha=0,1542 \mathrm{~nm}$ e filtro de níquel. As análises ocorreram entre os intervalos $5^{\circ} \mathrm{e} 80^{\circ}$ (2 Theta). As fases presentes foram analisadas no programa X - High Score Philips.

A natureza química da HAP sintetizada foi determinada pela análise de bandas características de grupos funcionais por meio do FTIR/ATR (NEXUS - 470 - FTIR, resolução 04, 32 varreduras).

A morfologia foi analisada por microscopia eletrônica de transmissão (TEM) (tensão 20-30 kV). A análise de espectroscopia por energia dispersiva (EDS) foi feita pelo equipamento TM $3000(15 \mathrm{kV})$.

\section{Adsorção de íons cobre $\left(\mathrm{Cu}^{+2}\right)$ em solução aquosa Influência do tempo (minutos)}

$\mathrm{A}$ adsorção de $\mathrm{Cu}^{+2} \mathrm{em}$ solução aquosa foi analisada em duas etapas, a primeira com análise em função do tempo, o sistema do estudo foi composto por $40 \mathrm{ml}$ de solução de $\mathrm{Cu}^{+2}$, em concentração de $90 \mathrm{mg} / \mathrm{L}$, e 0,04 g de HAP, sob agitação 200 rpm, por 24 horas. $\mathrm{O}$ pH foi ajustado para 5,0, com adição de $\mathrm{NaOH}$ $0,1 \mathrm{M}$. No final do experimento, as concentrações de metais foram analisadas (AAS, PERKIN ELMER ATOMIC ABSORPTION SPECTROMERT 3300).

A capacidade de remoção de $\mathrm{Cu}$ por HAP foi calculada utilizando-se as expressões matemáticas indicadas por Alhakawati e Banks (2004), Jang et al. (2008) e Mobasherpour, Salahi e Pazouki (2012) (Equação 2):

$q_{e}=\frac{c_{o}-c_{e q}}{M}$

Em que:

$\mathrm{q}_{\mathrm{e}}(\mathrm{mg} / \mathrm{g})=$ o carregamento;

$\mathrm{C}_{0}$ e $_{\text {eq }}=$ as concentrações inicial e final do metal em solução $(\mathrm{mg} / \mathrm{L})$, respectivamente;

$\mathrm{M}=\mathrm{a}$ massa do biossorvente $(\mathrm{g})$.

A taxa de remoção percentual (R\%) do $\mathrm{Cu}$ em solução foi calculada por meio da expressão (Equação 3), com as especificações previamente ditadas.

$R \%=\frac{\left(C_{0}-C_{e q}\right) x 100}{C_{\mathrm{o}}}$

\section{Factorial design}

A adsorção do $\mathrm{Cu}$ foi analisada por planejamento fatorial em função de 3 variáveis: $\mathrm{pH}(2,4$ e 6), concentração inicial de metal (50, 100 e $150 \mathrm{mg} / \mathrm{L})$ e massa de HAP adsorvente $(0,02 ; 0,04$ e $0,06 \mathrm{~g})$; e a interação multifatorial $(2 \times 3)$ foi realizada com 4 réplicas no ponto central.

Os sistemas foram compostos da solução de metal $(40 \mathrm{~mL})$ e das respectivas massas de HAP, sendo agitados a $200 \mathrm{rpm} / 6$ horas. $\mathrm{O} \mathrm{pH}$ foi ajustado com $\mathrm{NaOH} 0,1 \mathrm{M}$ ou $\mathrm{HNO}_{3}$ 0,1 M. No final do tempo esperado, a solução foi filtrada e o Cu residual em solução foi analisado por espectroscopia atômica em chama (AAS, PERKIN ELMER ATOMIC ABSORPTION SPECTROMERT 3300)

\section{RESULTADOS E DISCUSSÃO}

\section{Caracterização da hidroxiapatita}

A identificação cristalográfica da amostra de HAP foi determinada pela análise de XRD (Figura 1A), mostrando picos intensos e finos entre as regiões de $25^{\circ}<2 \theta$ $<50,0^{\circ}$. A análise da composição molecular foi realizada por FTIR (Figura 1B), em que se observam as seguintes vibrações típicas de HAP: $\mathrm{PO}_{4}^{-3}$ (560-610 e $1.000-1.100 \mathrm{~cm}^{-1}$ ) e OH- (636) (CENGIZ et al., 2008; NEIRA et al., 2009; SALIMI et al., 2012). O diâmetro das partículas de HAP foi analisado por meio de TEM, apresentando grãos circulares e tamanhos entre 40 e $290 \mathrm{~nm}$ (Figura 1C).

\section{Efeito do tempo de contato de hidroxiapatita na adsorção de $\mathrm{Cu}^{+2}$}

$\mathrm{O}$ efeito do tempo de contato para adsorção de $\mathrm{Cu}^{+2}$ pela HAP foi analisado e os resultados são apresentados na Figura 2. Observaram-se rápida adsorção e equilíbrio estabelecido em menos de 50 minutos.

Os resultados são similares a outros estudos com tempo de adsorção inferior a 100 minutos (CHEN et al., 2010; KUMAR et al., 2011). Ghandhi, Kousalya 


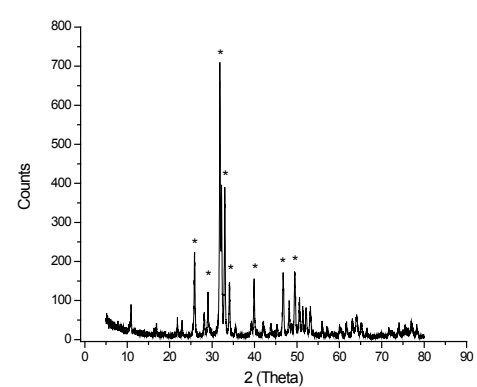

A

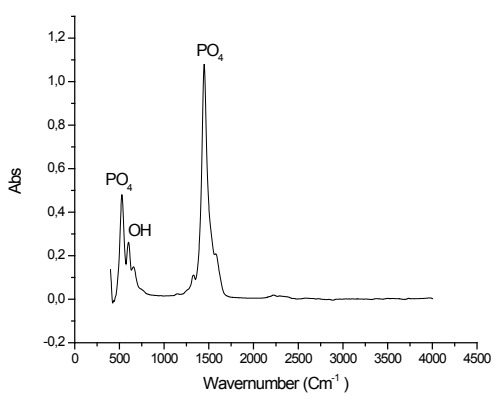

B

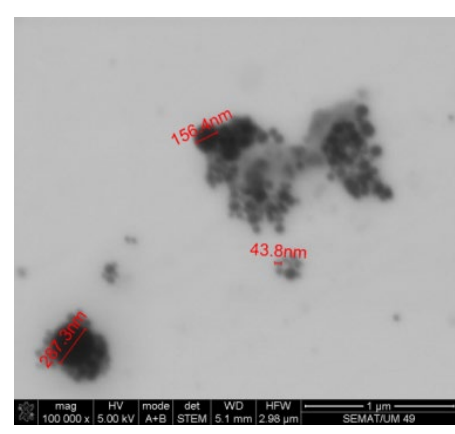

$\mathrm{C}$

Fonte: elaborada pelos autores.

Figura 1 - (A) Análise de difração de raios X, (B) espectroscopia no infravermelho por transformada de Fourier e (C) microscopia eletrônica de transmissão da hidroxiapatita produzida.

e Meenakshi (2011) analisaram a adsorção de Cu por HAP e composto HAP/ quitosana, e encontraram resultados equivalentes aos do presente estudo, com equilíbrio próximo a 30 minutos de exposição à solução metálica.

\section{Factorial design}

A Tabela 1 mostra o desenho experimental e os respectivos resultados analíticos, incluindo porcentagem de remoção, sendo observados alguns rendimentos superiores a $80 \%$. O modelo matemático de eficiência de remoção de $\mathrm{Cu}^{+2}$ é representado pela Equação 4:

$R \%=83,766+16,677 \mathrm{~A}-25,837 \mathrm{~B}+27,859 \mathrm{C}-0,336 \mathrm{AB}+1,159 \mathrm{AC}-26,078 \mathrm{BC}$

Uma análise de correlação foi realizada usando os dados obtidos da porcentagem de remoção (R\%) com ANOVA e respectivo valor $\mathrm{p}$, sendo demonstrada por meio do gráfico de Pareto (Figura 3A), com nível de confiança de $95 \%$. Os três fatores - pH da solução (1), massa adsorvente (3) e concentração inicial de metal (2) - e a interação $2 \times 3$ foram considerados importantes na remoção de $\mathrm{Cu}^{+2}$. A correlação entre os dados experimentais e preditivos foi obtida a partir dessas análises com $\mathrm{R}^{2}=$ 0,982 (Figura 3B).

\section{Análise de superfícies e espectroscopia por energia dispersiva}

A presença do $\mathrm{Cu}^{+2}$ adsorvido pelo HAP foi utilizada para construir os gráficos de superfície em função da R\% (Figuras 4A e 4B). A ocorrência do $\mathrm{Cu}^{+2}$ adsorvido pela HAP foi confirmada na análise do espectro EDS (Figura 4C).

A taxa percentual de remoção está diretamente correlacionada ao $\mathrm{pH}$ da solução, com maior rendimento entre pH 4,0 e 6,0, sendo considerado sendo considerado ideal para máxima remoção de $\mathrm{Cu}^{+2}$, conforme ilustram as regiões em vermelho (Figura 4). Em pH mais baixo, a competição entre o íon metálico $\left(\mathrm{Cu}^{+2}\right)$ e $\mathrm{H}^{+}$ocorre, dificultando a adsorção do metal (CHEN et al., 2010; KUMAR et al., 2011).

Em relação à concentração inicial do metal, a faixa ideal para melhor remoção seria entre 50 e $90 \mathrm{mg} / \mathrm{L}$. Isso ocorre porque haverá maior disponibilidade de sítios ativos de HAP para remover esse elemento. Já em concentrações mais

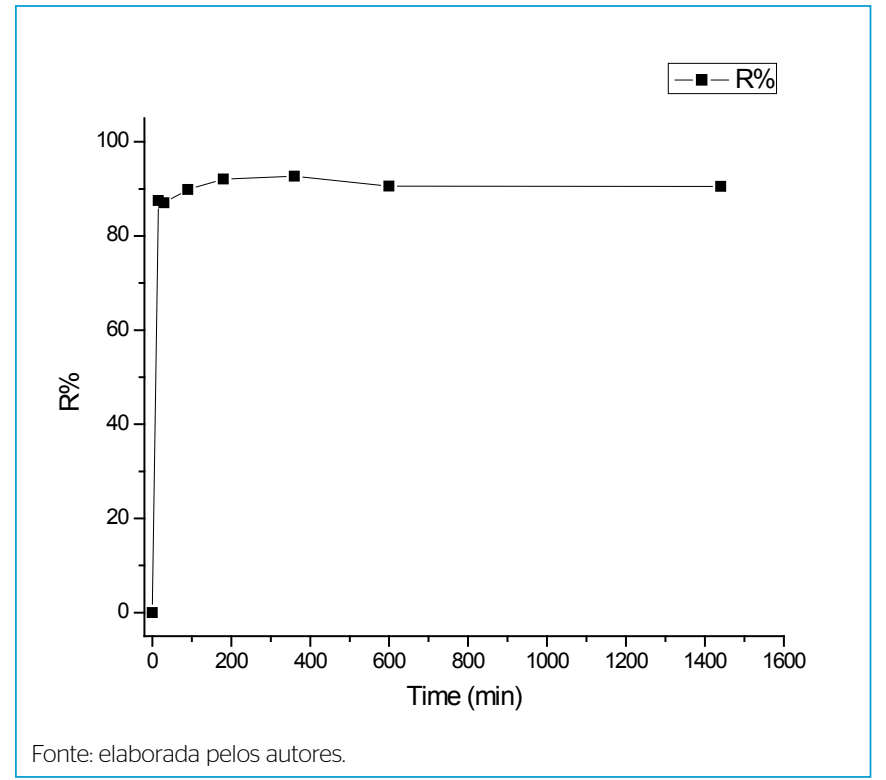

Figura 2 - Tempo de adsorção de Cu.

Tabela 1 - Matriz de projeto e os resultados do planejamento fatorial $(n=3)$.

\begin{tabular}{l|c|c|c|c} 
Experimentos & $\mathrm{pH}$ & $\mathrm{Ci}(\mathrm{mg} / \mathrm{L})$ & $\mathrm{HAP}(\mathrm{m})$ & $\mathrm{R} \%$ \\
\hline 1 & 0 & 0 & 0 & 84,42 \\
\hline 2 & + & - & - & 71,52 \\
\hline 3 & - & + & - & 40,96 \\
\hline 4 & 0 & - & + & 82,26 \\
\hline 5 & - & + & + & 42,21 \\
\hline 6 & + & + & - & 71,33 \\
\hline 7 & 0 & 0 & 0 & 85,33 \\
\hline 8 & 0 & 0 & 0 & 82,56 \\
\hline 9 & - & - & - & 39,81 \\
\hline 10 & + & + & + & 77,21 \\
\hline 11 & 0 & 0 & 0 & 82,75 \\
\hline 12 & - & - & + & 45,76 \\
\hline
\end{tabular}

Fonte: elaborada pelos autores. 


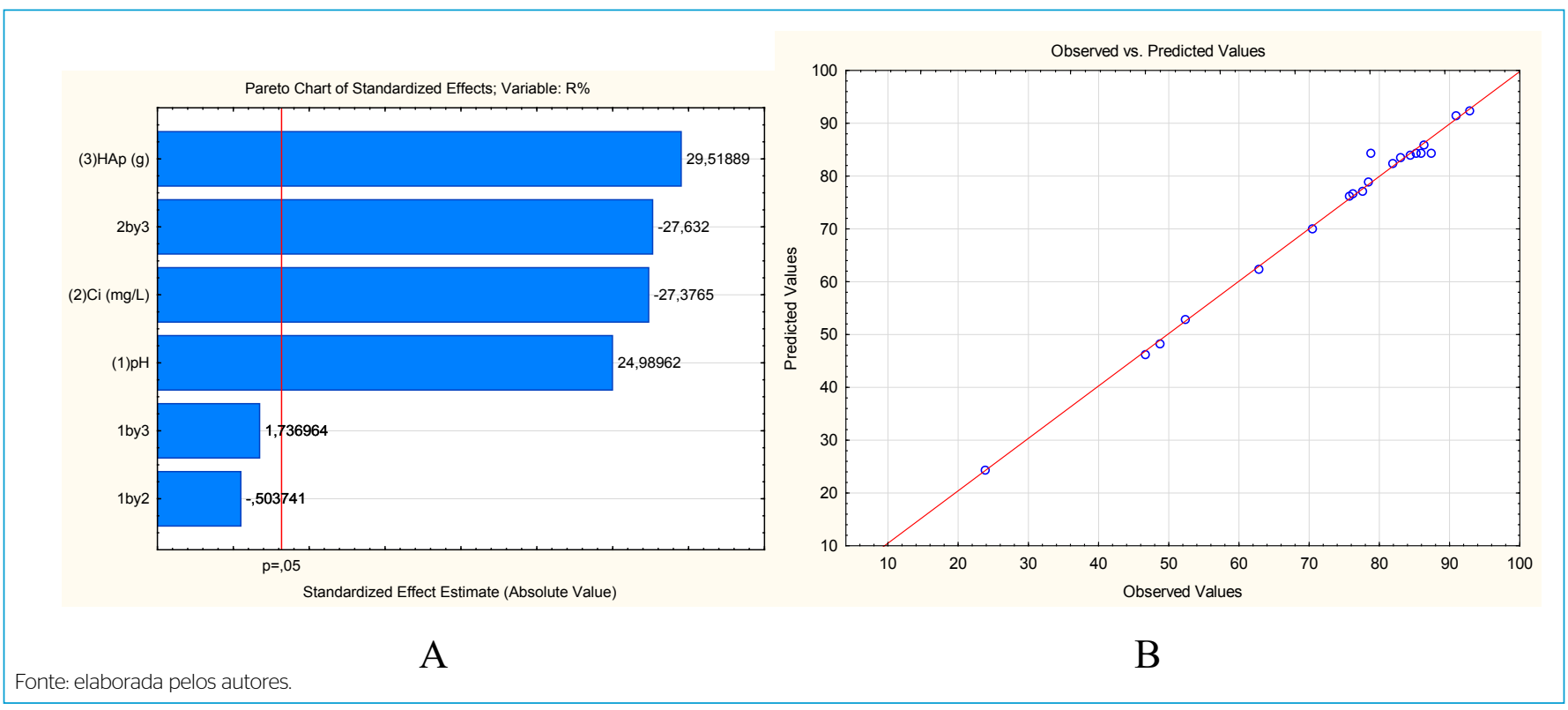

Figura 3 - (A) Resultados da análise de Pareto e (B) correlação de dados experimentais e preditivos.

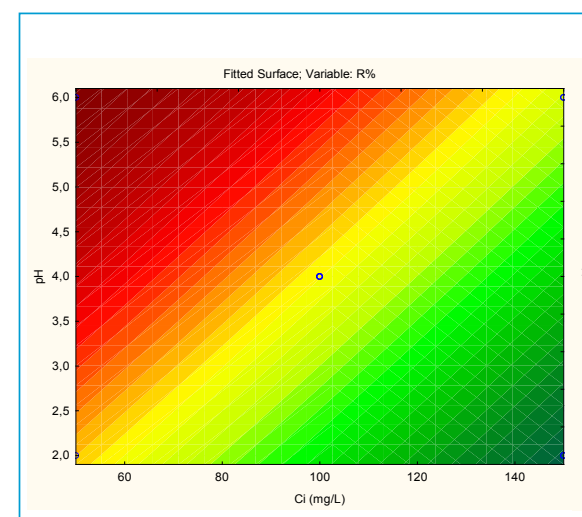

A

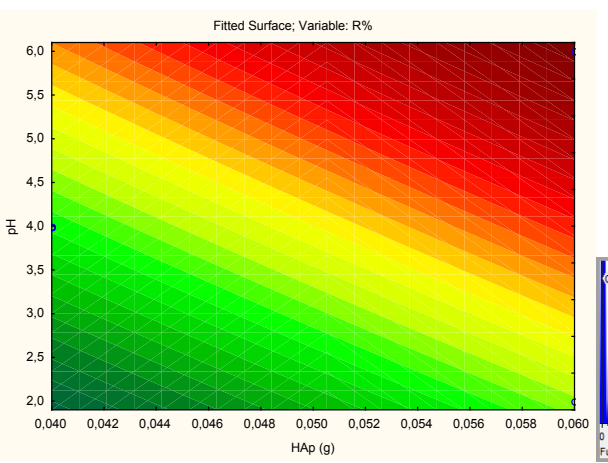

B

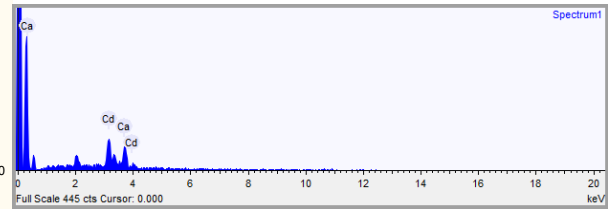

C

Fonte: elaborada pelos autores.

Figura 4 - (A e B) efeito do pH, do início da concentração (Ci) e da massa (HAP) na porcentagem de remoção (R\%); (C) espectroscopia por energia dispersiva - detecção do $\mathrm{Cu}^{+2}$ adsorvido na $\mathrm{HAP}>$ usado mostrando $\mathrm{Cu}^{+2}$ adsorvido.

altas esses locais são saturados mais rapidamente, reduzindo o rendimento da adsorção (CHEN et al., 2010; KUMAR et al., 2011).

A massa ideal de HAP a ser utilizada na adsorção foi entre 0,05 e 0,06 g, e justifica-se pela maior disponibilidade de sítios ativos insaturados para a absorção de íons $\mathrm{Cu}^{+2}$ em solução (KUMAR et al., 2011).

\section{CONCLUSÕES}

A análise dos efeitos de variáveis operacionais ( $\mathrm{pH}$, massa adsorvente e concentração inicial de metal) e suas interações na adsorção de íons $\mathrm{Cu}^{+2}$ por HAP foi realizada em planejamento fatorial $2 \times 3$.

A ANOVA mostrou que o pH da solução (1), a massa adsorvente (3), a concentração inicial de metal (2) e a interação $2 \times 3$ foram estatisticamente significantes. A eficiência máxima de remoção de $\mathrm{Cu}^{+2}$ obtida no procedimento de otimização foi de $85,33 \%$

A análise de correlação entre os fatores preditivos e experimentais foi analisada em $\mathrm{R}^{2}=0.982$. Assim, a HAP demonstrou baixo custo de produção e eficiência na remoção desse metal, sendo importante para a remediação ambiental em cenário de contaminação.

\section{CONTRIBUIÇõES DOS AUTORES}

Kramer, D. G.: Conceituação, Metodologia, Escrita - Primeira Redação, Investigação. Cavalcanti Junior, G. B: Curadoria de Dados, Análise Formal. Sousa, A. M: Conceituação, Análise Formal. Costa, J. B. S.: Metodologia, Investigação. 


\section{REFERÊNCIAS}

ALHAKAWATI, M.S.; BANKS, C.J. Removal of copper from aqueous solution by Ascophyllum nodosum. Journal of Environmental Management, v. 72, n. 4, p. 195-205, 2004. http://dx.doi.org/10.1016/j.jenvman.2004.04.010

CENGIZ, B.; GOKCE, Y.; YLDIZ, N.; AKTAS, Z.; CALIMI, A. Synthesis and characterization of hydroxyapatite nanoparticles. Colloids and Surfaces A: Physicochemical and Engineering Aspects, v. 322, n. 1-3, p. 29-33, 2008. https://doi.org/10.1016/j.colsurfa.2008.02.011

CHEN, H.; DAl, G.; ZHAO, J.; ZHONG, A.; WU, J.; YAN, H. Removal of copper(II) ions by a biosorbent-Cinnamomum camphora leaves powder. Journal of Hazardous Materials, v. 177, n. 1-3, p. 228-236, 2010. https://doi.org/10.1016/j.jhazmat.2009.12.022

CHENG, Z.; LIU, X.; HAN, M.; MA, W. Adsorption kinetic character of copper ions onto a modified chitosan transparent thin membrane from aqueous solution. Journal of Hazardous Materials, v. 182, n. 1-3, p. 408-415, 2010. https://doi.org/10.1016/j.jhazmat.2010.06.048

CÓRDOVA, J.C.; LÉON, A.M.G.; REGALADO, E.S.; GONZÁLEZ, M.N.S.; RAMÍREZ, T.L.; AVALOS, B.C.G.; MEDRANO, J.A.L. Experimental design for the optimization of copper biosorption from aqueous solution by Aspergillus terreus. Journal of Environmental Management, v. 95, supl., p. S77-S82, 2012. https://doi.org/10.1016/j.jenvman.2011.01.004

DA'NA, E; SAYARI, A. Optimization of copper removal efficiency by adsorption on amine-modified SBA-15: Experimental design methodology. Chemical Engineering Journal, v. 167, n. 1, p. 91-98, 2011. https://doi.org/10.1016/j.cej.2010.12.005

ESLAMI, H.; SOLATI-HASHJIN, M.; TAHRIRI, M.; BAKHSHI, F. Synthesis and characterization of nanocrystalline hydroxyapatite obtained by the wet chemical technique. Materials Science-Poland, v. 28, n. 1, p. 34, 2010.

FERNAME, F; MECHERRI, M.O.; SHARROCK, P.; FIALLO, M.; SIPOS, R. Hydroxyapatite interactions with copper complexes. Materials Science and Engineering C, v. 30, n. 7, p. 1060-1064, 2010. https://doi.org/10.1016/j. msec.2010.05.010

GHANDHI, M.R.; KOUSALYAB, G.N.; MENAKSHI, S. Removal of copper(II) using chitin/chitosan nano-hydroxyapatite composite. International Journal of Biological Macromolecules, v. 48, n. 1, p. 119-124, 2011. https://doi. org/10.1016/.i.jbiomac.2010.10.009

JANG, S.H.; MIN, B.G.; JEONG, Y.G.; LYOO, W.S.; LEE, S.C. Removal of lead ions in aqueous solution by hydroxyapatite/polyurethane composites foams, Journal of Hazardous Materials, v. 152, n. 3, p. 1285-1292, 2008. https://doi. org/10.1016/j.jhazmat.2007.08.003

KUMAR, P.S.; RAMALINGAM, S.; SATYASELVABALA, V.; KIRUPHA, S.D.; SIVANESAN, S. Removal of copper(II) ions from aqueous solution by adsorption using cashew nut shell. Desalination, v. 266, n. 1-3, p. 63-71, 2011. https://doi.org/10.1016/j.desal.2010.08.003 kyzas, G.Z; bikiari, D.N.; Kostoglou, M.; Lazaridis, N K. Copper removal from aqueous systems with coffee wastes as low-cost materials. Web of Conferences, v. 1, p. 25, 2013. https://doi.org/10.1051/e3sconf/20130125004

LIANG, W.; ZHAN, L.; PIAO, L.; RUSSEL, C. Lead and copper removal from aqueous solutions by porous glass derived calcium hydroxyapatite Materials Science and Engineering B, v. 176, n. 13, p. 1010-1014, 2011. https:// doi.org/10.1016/j.mseb.2011.05.036

MOBASHERPOUR, I.; SALAHI, M.; PAZOUKI, M. Comparative of the removal of $\mathrm{Pb} 2+, \mathrm{Cd} 2+$ and $\mathrm{Ni2}+$ by nano crystallite hydroxyapatite from aqueous solutions: Adsorption isotherm study. Arabian Journal of Chemistry, v. 5, n. 4, p. 439-446, 2012. https://doi.org/10.1016/j.arabjc.2010.12.022

MONDAL, S.; MONDAL, B.; DEY, A.; MUKHOPADHYAY, S.S. Studies on processing and characterization of hydroxyapatite biomaterials from different bio wastes. Journal of Minerals \& Materials Characterization \& Engineering, v. 11, n. 1, p. 55-67, 2012.

NAYAK, Y. Hydroxyapatite - TZP composites: processing, mechanical properties, microstructure and in vitro bioactivity. 196f. Thesis (Doctorate) - Department of Ceramic Engineering, National Institute of Technology, Rourkela, 2010

NEIRA, I.; KOLEN'KO, Y. V.; LEBEDEV, O. I.; TENDELOO, G. V.; GUPTA, H. S.; GUITIÁN, F.; YOSHIMURA, M. An effective morphology control of hydroxyapatite crystals via hydrothermal synthesis. Crystal Growth \& Design, v. 9, n. 1, p. 466-474, 2009. https://doi.org/10.1021/cg800738a

Rosskopfova, O.; Galambos, M.; OMETÁKOVÁ, J.; CAPLOVICOVÁ, M.; RAJEC, P. Study of sorption processes of copper on synthetic hydroxyapatite. Journal Radioanal Nucl Chemistry, v. 293, p. 641-647, 2012. https://doi. org/10.1007/s10967-012-1711-4

SALIMI, M.S.N.; BRINDSON, R.H.; GROVER, L.M.; LEEKE, G.A. Effect of processing conditions on the formation of hydroxyapatite nanoparticles. Powder Technology, v. 218, p. 109-118, 2012. https://doi.org/10.1016/j. powtec.2011.11.049

SANTOS, V.C.G.; SOUZA, J.V.T.M; TARLEY, C.R.T.; CAETANO, J.; DRAGUNSKI, D.C. Copper ions adsorption from aqueous medium using the biosorbent sugarcane bagasse in natura and chemically modified. Water, Air, \& Soil Pollution, v. 216, p. 351-359, 2011. https://doi.org/10.1007/ s11270-010-0537-3

SHENG, G.; LI, J.; SHAO, D.; HU, J.; CHEN, C.; CHEN, Y.; WANG, X. Adsorption of copper(II) on multiwalled carbon nanotubes in the absence and presence of humic or fulvic acids. Journal of Hazardous Materials, v. 178, n. 1-3, p. 333 340, 2010. https://doi.org/10.1016/j.jhazmat.2010.01.084 